\title{
Remote Lab Experiments in Electronics for Use and Reuse
}

\author{
http://dx.doi.org/10.3991/ijim.v9i2.4346 \\ T. Zimmer, M. Billaud, M. Pic, D. Geoffroy \\ University of Bordeaux, Talence, France
}

\begin{abstract}
This paper presents a new approach for the use of an already existing remote lab in the field of electrical engineering education. About 70 experiments representing a wide domain of electronic functions are easily accessible and can be incorporated into an existing teaching environment by an easy copy/paste action of a dedicated URL. The corresponding database structure is explained and a typical result is given to highlight the procedure to follow.
\end{abstract}

Index Terms-Laboratory experiments, Remote labs, Reuse of remote labs, Engineering education

\section{INTRODUCTION}

In sciences education, experimental methods are crucial for understanding basic concepts students face during practical sessions. Moreover, experimental methods give students an opportunity to develop their perceptions and autonomy and, finally, to increase their interest in a given topic. In the electrical engineering (EE) curriculum, comprehension of electrical functions realized through simple circuits is a key for successful achievement of educational goals. Physical realization of the function leads to nonidealities that must be quantified by electrical measurements [1]. Furthermore, limitations of the measurement condition and the measurement resolution have to be analyzed [2]. A remote laboratory for characterizing electronic circuits has many advantages: it allows sharing highquality instruments and full-custom circuits that cannot be duplicated in several labs for cost reasons. Net-based access allows the users to launch experiments and analyze the results any place as often as they want [3]. The remote laboratories' usefulness, acceptance and learning results have already been proven [4] and consolidated studies are in progress [5].

Setting up a remote lab demands not only knowledge in electrical engineering, but also knowledge in computer science, making the whole procedure quite complex. Many remote labs already exist and are referenced (e.g., on the lab2go platform) [6] or can be available through remote lab networks [7]. The reuse of remote labs is not straightforward. From a pedagogical point of view, the associated learning content rarely fits with what is necessary for a given lecture; in addition, the "overhead" activities such as enrollment in an existing remote lab, authentication and reservation for their employment for a given time slot very often represent very often a serious obstacle for wide use/reuse of existing remote labs.

In this paper we report a new approach for reusing remote labs. First, we present a library in which about 70 different experiments are listed and described. These experiments cover about $80 \%$ to $90 \%$ of the electronic functions that are commonly investigated during electrical engineering education up to the bachelor's level. Next, we present the organization of the database where all information about these circuits can be found. Finally, we show how each professor/teacher/instructor can access these experiments by simply copying/pasting one single hyperlink and integrating this link inside his personal teaching material. Of course, the teaching material must be available online (e.g., using OER or an LMS such as Moodle).

The paper is organized as follows: Section 2 gives an overview of the 70 experiments that can be freely used; Section 3 describes the database architecture illustrating the different experiments; Section 4 develops how a professor/teacher/instructor can integrate these remote experiments into his own learning environment by a simple copy/past operation; and finally, a conclusion is given in Section 5.

\section{AVAILABLE EXPERIMENTS}

The different electronic circuits that are usually examined during electrical engineering education can be classified into different categories:

\section{A. Passive Electronic Circuits}

In the easiest form, a passive circuit is only a dipole such as a resistor, a capacitor, or an inductor. Combining $\mathrm{C}$ and $\mathrm{R}$ components can form a passive low-pass or highpass filter, and by adding an inductor, the three components can form a resonant circuit. All these different kinds of circuits are available for frequency domain measurement (e.g., Bode-diagram of a filter) as well as in the time domain thanks to the employment of a function generator and an oscilloscope.

\section{B. Diodes And Transistors Based Circuits}

Diodes and transistors form the foundation of any electronic circuit. Their investigations in many configurations are available:

a. Diode half wave rectifier

b. Common emitter amplifier (in different configurations)

c. Emitter follower

d. Common base amplifier

e. PNP-common emitter amplifier

f. Push-Pull output stage

g. The transistor as a switch

Most of the circuits can be investigated in both time domain and frequency domain. 


\section{REMOTE LAB EXPERIMENTS IN ELECTRONICS FOR USE AND REUSE}

\section{Emitter Coupled Pair Circuits}

The next class concerns the differential amplifier. For the realization of this function, different configurations are possible and can be examined:

a. Differential amplifier with long tail current source

b. Differential amplifier with active current source

c. Differential amplifier with passive load

d. Differential amplifier with active load

To get the whole picture of the differential amplifier, it can be investigated in differential mode and in common mode.

\section{Op-Amp Based Circuits}

Operational amplifiers are basic building blocks for many electronic functions such as

a. Comparator with Op-Amp (741), Slewrate

b. Comparator with Op-Amp (TL081), Slewrate

c. Op-Amp Amplifier in closed loop, input impedance investigation

d. Active low pass filter

e. Comparator (inverted)

f. Comparator with a threshold

g. Derivator

h. Integrator

i. Inverter, voltage gain $=2$

j. Inverter, voltage gain $=10$

$\mathrm{k}$. Voltage follower

1. Non-Inverter

$\mathrm{m}$. Non-Inverter, voltage gain $=10$

n. Non-Inverter, voltage gain $\sim 50$

o. Non-Inverter, Output current limits investigation

p. Op-Amp with positive feedback

q. Schmitt-Trigger

All of these circuits can be investigated through the remote lab.

\section{ARCHITECTURE OF THE DATABASE}

For the creation of the database, we used SQLite through the SQL language. Furthermore, thanks to the PHP language and the function jQuery from Javascript we generated a table that shows information about the circuits on different levels:

The first level contains the basic information such as (see Fig.1):

a. Reference: a reference code for identifying the experiment

b. Title: a first indication about the experiment

c. Details: detailed information about the experiment

d. Component : list of the components used

e. Liens : hypertext link for access to the experiment in a different learning environment through copy/paste

The second level contains detailed information about each experiment; it can be accessed by clicking on the (i)icon (see Fig.2):

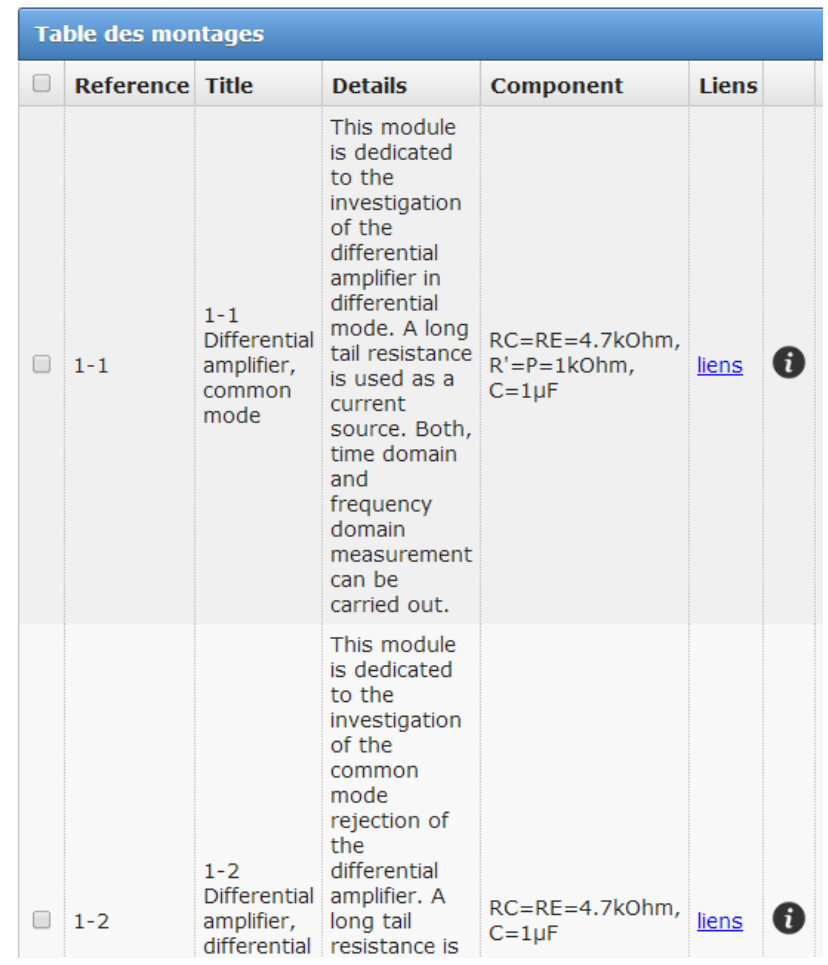

Figure 1. First level of information for the experiments

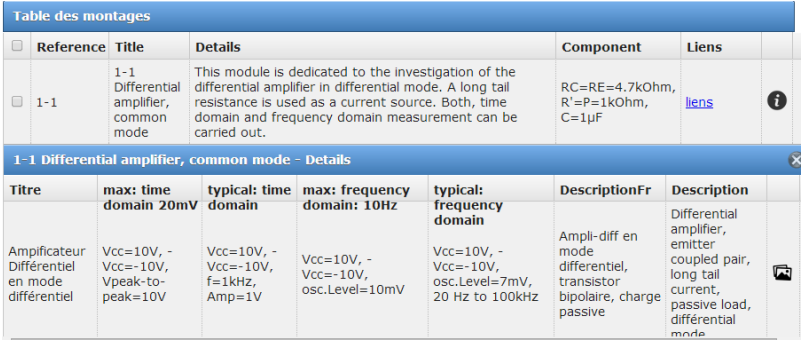

Figure 2. Second level of information for the experiments

a. Title

b. Maximum settings for time-domain measurements

c. Typical settings for time-domain measurements

d. Maximum settings for frequency-domain measurements

e. Typical settings for frequency-domain measurements

f. A description in French

g. A description in English

h. A picture-icon

The third level gives additional information for each experiment (see Fig.3):

Image 1: the circuit diagram

Image 2: a photograph of the module

Image 3: a typical measurement result in time domain

Image 4: a typical measurement result in frequency domain

Furthermore, an administrator interface has been set up that permits users to change/add/delete all the information that is in the database on the fly. Figure 4 shows the interface dedicated to adding another experiment. 


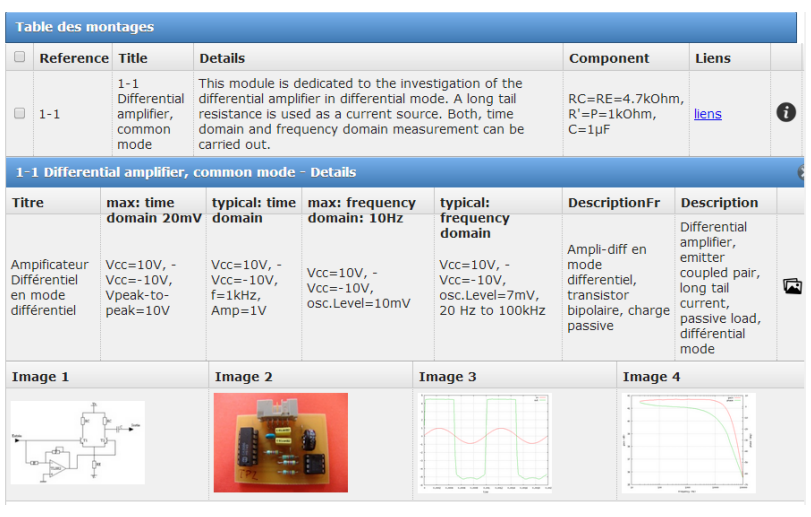

Figure 3. Third level of information for the experiments

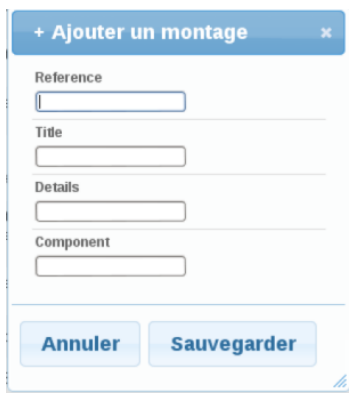

Figure 4. User interface for administration of the data base

\section{USING THE EXPERIMENTS}

This section describes how to incorporate a remote experiment into a given learning environment. In the first step, we assume the professor/teacher/instructor has made his choice from among the different experiments he wants to use. Next he clicks on the hyperlink, called "liens," seen in the first table (see Fig.1). When doing so, a window pops up indicating the link that has to be copied and pasted into the teacher's document (see Fig.5).

http://elab.creea.u-bordeaux1.fr/ELAB/docs/manip.php?experiment $=X X X X$

Figure 5. Typical link to copy/paste

The name " $\mathrm{X}$ " is different for each experiment and the user does not have to worry about it.

Now, when the student works with the teacher's learning material and clicks on the link that has been incorporated by the teacher into those learning documents, a window opens asking the students to specify the stimuli (settings for the measurement instruments, such as voltage level, to apply the frequency, the wave form and so on). An example is shown in Fig.6. These data are then transferred to our remote lab (www.real-lab.org), and the measurement is executed by applying the above stimuli.

When the measurement is completed, the results are transferred to the student's computer and are displayed. (An example is shown in Fig.7) Now the student can exploit the measured data and respond to the teacher's specific questions.

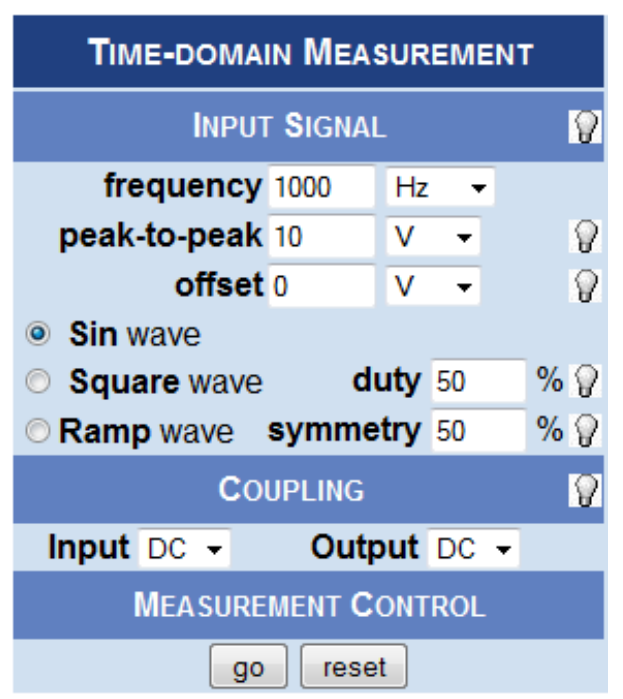

Figure 6. Measurement interface specifying the settings

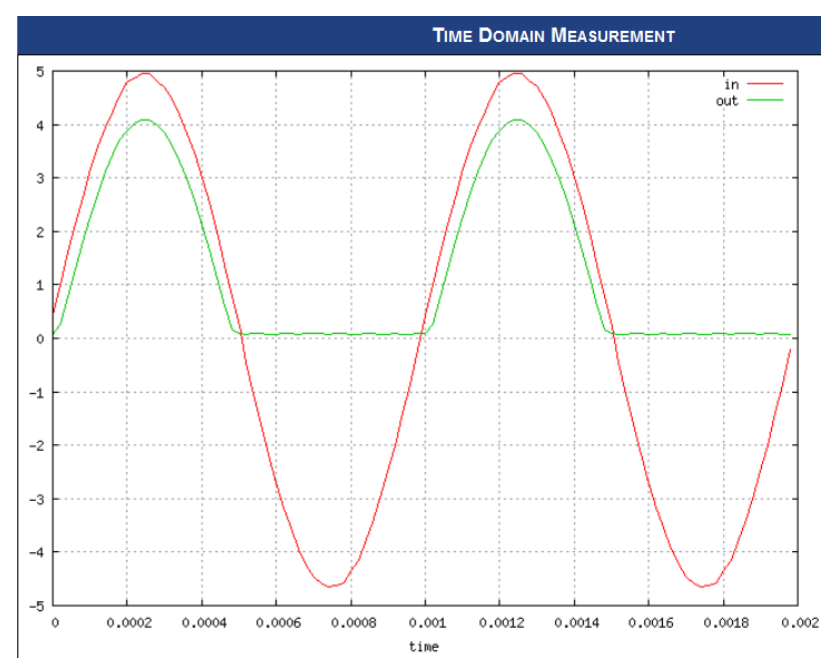

Figure 7. An example of measurement results

\section{CONCLUSION}

In this paper we presented a new approach about how to use an already existing remote lab in the field of electrical engineering education. Approximately 70 experiments describing a wide domain of electronic functions are easily reachable and can be incorporated in any existing remotely accessible teaching environment by a straightforward copy/paste action. An overview of the 70 experiments has been given and the corresponding database structure has been explained. In a cookbook manner, the procedure about how a professor/teacher/instructor can simply incorporate a given experiment in his private teaching documents is explained. The main advantage compared to existing solutions is that in the proposed approach, the instructor/teacher/professor has maximum freedom in constructing his course and can easily enhance the course content by adding real lab modules, so the students will benefit from the real world applications. 


\section{REFERENCES}

[1] M Billaud, T Zimmer, D Geoffroy, Y Danto, H Effinger, W Seifert, J Martinez, F Gomez, "Real measures, virtual instruments," Proceedings of the Fourth IEEE International Caracas Conference on Devices, Circuits and Systems, I023-1-I023-4, 2002

[2] T Zimmer, M Billaud, D Geoffroy, "Remote laboratory for electrical engineering education," International Journal of Online Engineering 2 (3), 2006

[3] N Lewis, M Billaud, D Geoffroy, P Cazenave, T Zimmer, "A distance measurement platform dedicated to electrical engineering," Learning Technologies, IEEE Transactions on 2 (4), 312319, 2009 http://dx.doi.org/10.1109/TLT.2009.45

[4] D Lang, C Mengelkamp, RS Jäger, D Geoffroy, M Billaud, T Zimmer, "Pedagogical evaluation of remote laboratories in eMerge project," European Journal of Engineering Education 32 (1), 57-72 http://dx.doi.org/10.1080/03043790601055626

[5] T. Tsiatsos, S. Douka, T. Zimmer, D. Geoffroy, "Evaluation plan of a network of remote labs in the Maghrebian countries", Conference REV 2014, February 26-28, 2014, Porto, Portugal http://dx.doi.org/10.1109/REV.2014.6784255

[6] www.lab2go.net

[7] T. Zimmer, D. Geoffroy, A. Pester, R. Oros, T. Tsiatsos, S. Douka, " eSience: Setting up a network of remote labs in the Maghrebian countries" iCEER International Conference on Engineering Education and Research, Marrakesh, July 1st-5th, 2013, Morocco

\section{AUTHORS}

T. Zimmer is with the Department of Applied Physics and Measurement Engineering at the Institute of Technology at the University of Bordeaux. He received a M.Sc. in Physics from the University of Würzburg, Germany, in 1989 and a Ph.D. in Electronics from the University Bordeaux 1, Talence, France, in 1992. From 1989 to 1990, he was with the Fraunhofer Institute, Erlangen, Germany. Since 1992, he has been with the IMS Institute, Talence, France. Since 2003, he has been a professor at the University of Bordeaux. At the IMS lab, he is the leader of the research group "Nanoelectronics." $\mathrm{He}$ is a cofounder of XMOD Technologies and a senior member of IEEE. He has published more than 200 peer-reviewed scientific articles, 1 book and contributed to 8 book-chapters. His education interests concern multimedia, open and distance learning and the implementation of ICT (information and communication technologies) in lab courses. In particular, he was the coordinator of 3 European projects dedicated to remote labs.

M. Billaud is with the Computer Science Department at the Institute of Technology at the University of Bordeaux, France. He received a PhD in Applied Mathematics (computer science specialty) from the University of Bordeaux 1, France, in 1985. He joined the Institut Universitaire de Technologie de l'Université Bordeaux 1, where he teaches mainly about operating systems, programming, and networks. His research interests include formal semantics, graph rewriting, and e-learning. He has been involved in various e-learning projects in Bordeaux since 2000.

M. Pic was a third year student when doing this work at the Computer Science Department at the Institute of Technology at the University of Bordeaux, France.

D. Geoffroy is with the Electrical Engineering Department at the University of Bordeaux, France. He received an MSc in Applied Physics from the University of Orsay, Paris, in 1980 . He is currently an associate professor in the Department of Electrical and Electronic Engineering at the University of Bordeaux and is also the head of the Pedagogical Lab Center of this department. His special interests include multimedia, open and distance learning, and the implementation of information and communication technologies (ICT) in practical courses.

This project has been funded with support from the European Commission. This publication reflects the views of only the authors, and the Commission cannot be held responsible for any use which may be made of the information contained therein.

Submitted, December, 19, 2014. Published as resubmitted by the authors on May, 16, 2015. 